\title{
Diastolic murmur of coronary artery stenosis
}

\author{
J. F. Sangster ${ }^{1}$ and C. M. Oakley \\ From the Department of Medicine (Clinical Cardiology), Royal Postgraduate Medical School \\ and Hammersmith Hospital, London
}

Three patients with a diastolic murmur associated with coronary artery stenosis are described. The source of the murmur was conclusively proved in the first case, and in the other patients the murmur and coronary angiographic findings were identical. The characteristics of the murmur and its mechanism of production are described. Since the three patients were all seen within a six-month period, this sign is probably not a rarity.

It is concluded that the diastolic murmur is an important indicator of non-critical stenosis of the left anterior descending coronary artery.

Physical findings in patients with coronary artery disease have been overshadowed by more spectacular diagnostic methods, and there has been little recent emphasis on physical examination (Cheng, I970a). A diastolic murmur associated with coronary artery stenosis is uncommon. A careful study of diastolic sounds in 93 patients with proven coronary artery disease (Cohn et al., 197I) demonstrated no diastolic murmurs. The few reported cases have received little attention, but in most the association of the murmur and coronary stenosis was presumptive only.

This study describes three patients in whom a diastolic murmur was associated with coronary artery stenosis. The diagnosis was conclusively proved in the first case, and the other cases were found within a further six months. The features of the murmur are described and the mechanism of its production discussed.

\section{Case I}

\section{Case reports}

A 35-year-old bricklayer was admitted to Hammersmith Hospital in July 197I for investigation of incapacitating angina pectoris. Apart from a soft apical fourth heart sound, examination was normal. No cardiac murmurs were present. Chest $x$-ray, electrocardiogram, and glucose tolerance were normal, but serum cholesterol and triglyceride levels were high. At cardiac catheterization, the intracardiac pressures and left ventricular angiogram were normal. Coronary angiography had to be aban-

Received 26 March 1973.

${ }^{1}$ National Heart Foundation of Australia Overseas Clinical Fellow. Present address: Cardiac Clinic, Royal Adelaide Hospital, South Australia. doned because of severe pain, hypotension, and electrocardiographic changes following the first injection into the left coronary artery. There was no subsequent evidence of myocardial infarction, and he was discharged for a period of medical therapy.

Nine months later he was readmitted after three prolonged attacks of rest pain and the development of electrocardiographic changes of inferior subendocardial infarction with transient Wenkebach atrioventricular block. For the first time, a high pitched early diastolic murmur was noted, crescendo-decrescendo in character, grade $2 / 4$ in intensity, and localized to a small area over the third left intercostal space, $4 \mathrm{~cm}$ from the midsternal line. The murmur was most easily heard with the patient sitting upright, and was confirmed by phonocardiography (Fig. I). It was felt to be due to coronary artery stenosis. A late systolic click was also audible.

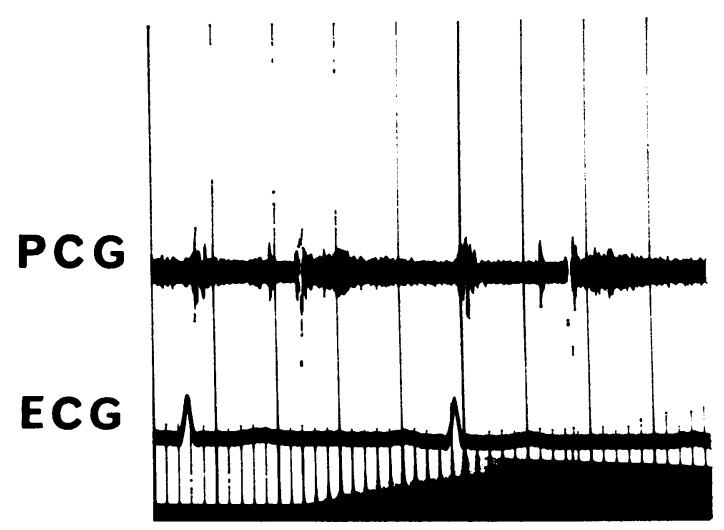

FIG. I Phonocardiogram showing diastolic murmur (and a late systolic click). 


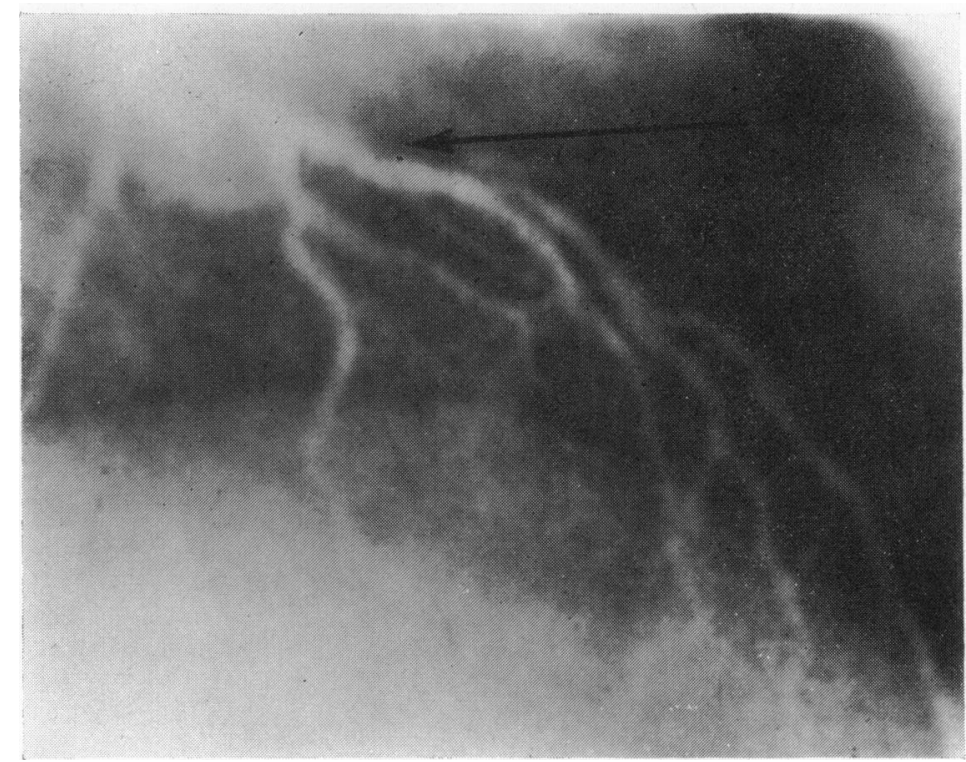

FIG. 2 Coronary arteriogram showing left anterior descending stenosis (arrowed).

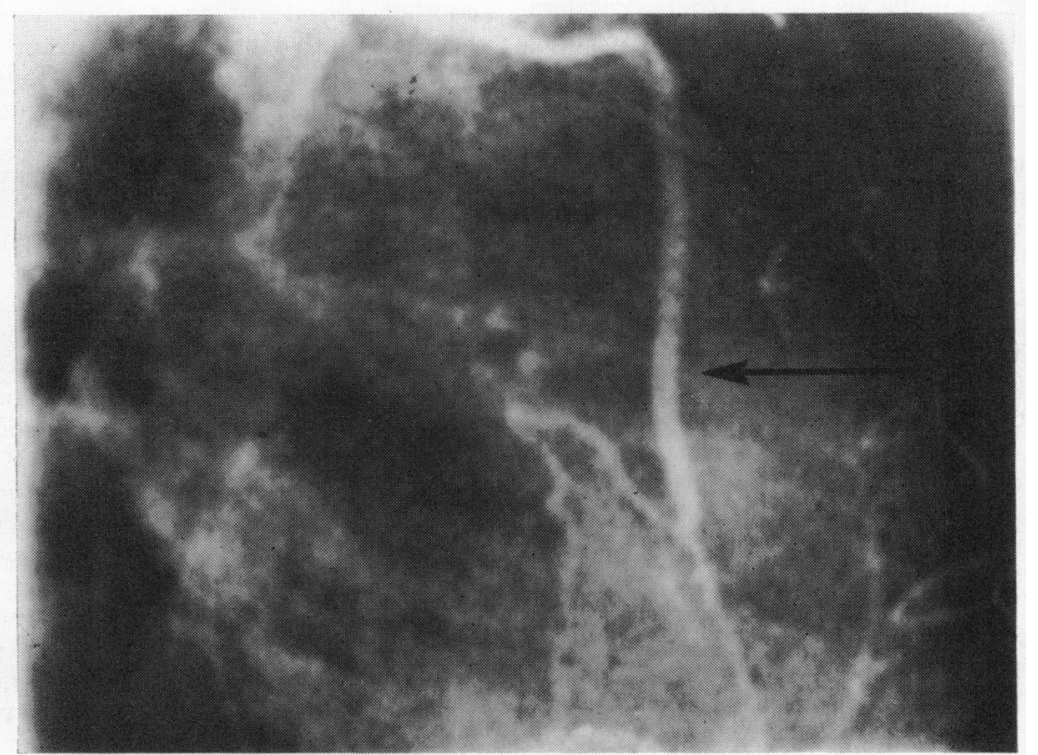

FIG. 3 Graft injection showing widely patent graft (arrowed) with filling of left anterior descending vessel. 


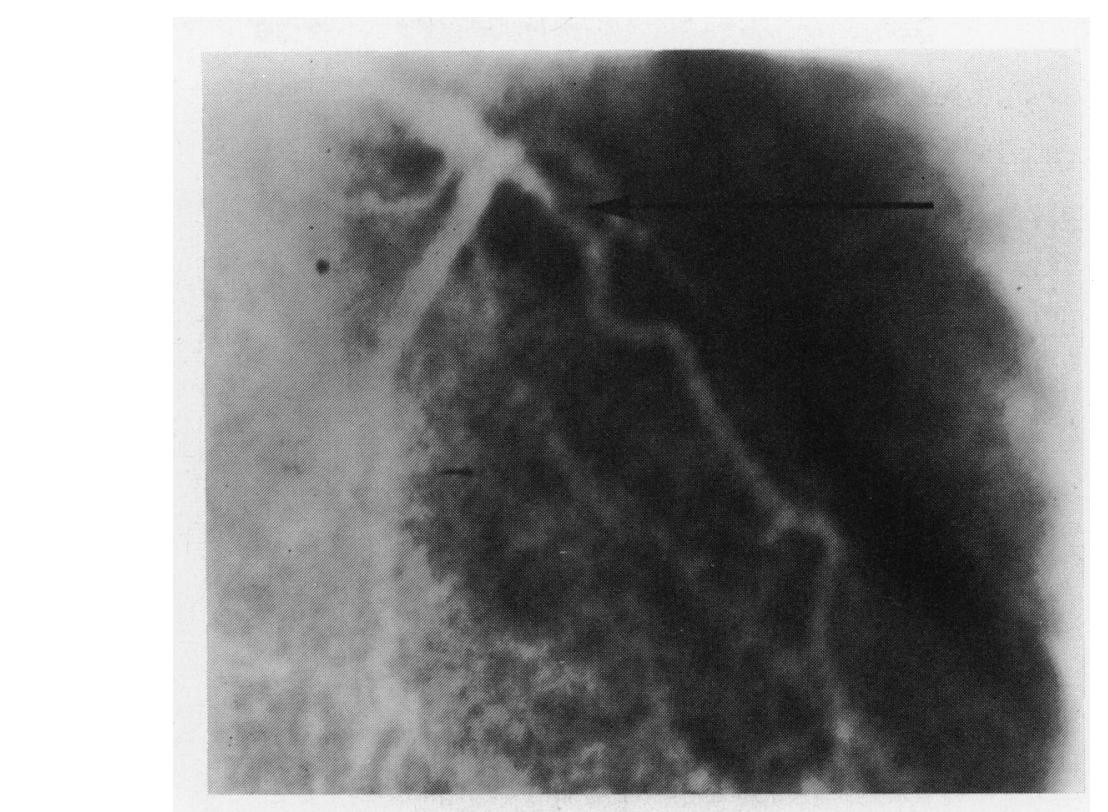

FIG. 4 Postoperative coronary angiogram showing persisting left anterior descending stenosis (arrowed).

The patient recovered uneventfully, but his angina recurred on ambulation. The murmur remained. Coronary angiography was carried out without complications and showed total occlusion of the proximal right coronary artery with retrograde filling via the left coronary system. In the proximal third of the left anterior descending coronary artery, corresponding closely to the site of the murmur, was a 50 per cent stenosis (Fig. 2). Aortic root injection showed a normal aortic valve without regurgitation. At operation a diastolic thrill was readily palpable over the left anterior descending vessel, immediately distal to the stenosed segment. The rest of the heart appeared normal. The right coronary artery was found to be unsuitable for any grafting procedure. A single saphenous vein graft was therefore placed from aorta to the left anterior descending vessel, distal to the stenosis, and good flow obtained. The postoperative course was uneventful, and the murmur was no longer audible.

Unfortunately the angina recurred when the patient became ambulant again. Further coronary angiography was carried out five months after operation. This showed a widely patent graft filling the left anterior descending vessel (Fig. 3). The proximal segment filled retrogradely and the stenosis showed little change from the preoperative appearances (Fig. 4). The patient's continued angina was therefore attributed to failure to revascularize the territory of the right coronary artery. As this vessel was known to be too diseased to accept a graft and the anterior descending graft was perfusing a large territory, further operation was felt to be inadvisable. He has since improved with increased medical therapy.

\section{Case 2}

A 32-year-old solicitor suffered extensive inferoapical myocardial infarction in July 1969, but made a good recovery and was able to resume work. Two years later another episode of chest pain caused readmission to Hammersmith Hospital, but further myocardial infarction was not confirmed. Apart from an apical fourth sound, there were no abnormal heart sounds or murmurs. Hypercholesterolaemia was present. Coronary angiography showed a normal right coronary artery, occlusion of a major muscular branch of the circumflex vessel, and a moderate left anterior descending stenosis. He made an uneventful recovery and was started on medical therapy.

One year later he was admitted with further atypical chest pain, but on this occasion a diastolic murmur was readily heard. As in the previous patient, it was a highpitched, early, crescendo-decrescendo murmur of low intensity, situated over the third left intercostal space, $3 \mathrm{~cm}$ from the midsternal line. Myocardial infarction was not confirmed by electrocardiographic or enzyme changes and coronary angiography was repeated. This showed very slight increase in the left anterior descending stenosis and an area of apical akinesia, but there had been little significant change since the previous examination. Aortic root injection showed no reflux. Medical measures were continued, and the patient discharged without symptoms.

\section{Case 3}

A 62-year-old wood machinist was referred to outpatients 
with a three-year history of angina pectoris, increasing in severity, and the recent development of a diastolic murmur. Auscultation showed an apical fourth heart sound and confirmed the diastolic murmur. It was high pitched, grade $2 / 4$ in intensity, sharply localized to a small area over the third left intercostal space $4 \mathrm{~cm}$ from the midline, and best heard with the patient sitting up.

One month later he was admitted for full investigation. During that period he had had several prolonged attacks of chest pain, though the electrocardiogram showed no new changes. The murmur was no longer audible. Coronary angiography showed a complete obstruction of the left anterior descending vessel, together with diffuse disease in both right and left circumflex vessels. Some ventricular dyskinesia was present. Aortography showed no aortic regurgitation. The diffuse disease was felt to make him a poor candidate for successful bypass grafting, but in view of his continuing severe rest pain surgery was carried out in the hope that at least the left anterior descending occlusion was of recent origin and could be bypassed. This vessel was found to be completely occluded by atheromatous material in its proximal segment but a graft could be anastomosed from the aorta to the vessel distal to the occlusion. Graft flow was considered satisfactory. Unfortunately he developed a severe hemiplegia with signs of brain stem damage and died on the fifth postoperative day. Necropsy confirmed the operative findings of a total occlusion of the left anterior descending vessel by atheromatous material and thrombus.

\section{Discussion}

Only six cases of a diastolic murmur thought to be due to coronary artery stenosis have been reported, and in most the relation was open to doubt. In all, the murmur was thought to arise from the left anterior descending artery. The first report (Dock and Zoneraich, 1967) described a patient with a similar diastolic murmur and concluded it arose from the only abnormality at necropsy, a stenosed coronary artery. The authors also alluded to a second case but gave no details. Three other patients have been described (Fearon et al., 1968; Cheng, 1970b) in whom diastolic murmurs were thought to be due to angiographically demonstrated coronary stenoses, the murmurs disappearing with the development of myocardial infarction. It was assumed that the murmur disappeared when a partial occlusion became complete, giving rise to infarction. No necropsy was carried out on the patients who died, while the survivor was not restudied. The most fully documentated case report (Lund-Larsen, 1967) described the murmur, showed a stenosis at angiography, saw the murmur disappear with infarction, and found acute thrombotic occlusion at the site of the previous stenosis at necropsy.

There can be no doubt of the direct association between the coronary stenosis and the murmur in our first case. Aortic regurgitation was excluded by aortography; coronary angiography showed a lesion corresponding closely to the anatomical site of the murmur; a diastolic thrill was readily palpable at operation. The disappearance of the murmur after operation was not surprising. It could have resulted from complete occlusion developing in the stenosed segment, but repeat angiography showed both graft and anterior descending vessel to be patent. The graft, by equalizing the pressures on either side of the coronary stenosis, abolished the flow across it so that the murmur disappeared.

The two subsequent cases both present adequate evidence to support the diagnosis of a coronary stenosis murmur. The physical characteristics of the murmur were exactly similar and aortic incompetence was excluded by aortography. Case 2 developed the murmur while under regular observation, suggesting that the slight increase in left anterior descending narrowing was enough to cause a critical change in flow patterns and produce the murmur. The murmur of the third case disappeared with the advent of preinfarction angina, probably because of the development of complete obstruction in a previously stenosed anterior descending vessel.

The characteristics of the murmur in our cases were similar to those described in the earlier reports. Blood flow in the left coronary system is mainly diastolic (Gregg, 1950) due to the 'throttling' action of left ventricular contraction on the smaller coronary branches (Sabiston and Gregg, 1957). This systolic compression is exaggerated if a proximal stenosis reduces the distal intraluminal pressure. The systolic component of flow may be increased on exercise and approach the diastolic flow rate (Gregg, 1967). Thus at rest the murmur produced by flow through a stenosed artery of the left coronary system is diastolic. Because of its low intensity, the murmur is sharply localized over areas where a stenosed vessel is close to the chest wall. Therefore it is unlikely that a murmur will be heard except with left anterior descending coronary stenosis. The murmur is superficial and high pitched, and has a crescendodecrescendo quality, corresponding with observations of maximal coronary flow in the second quarter of diastole (Gregg, I950). It is easily missed, and this may provide a partial explanation of its rarity (Cheng, I970b). In our cases, careful auscultation was needed to confirm the continuing presence of the murmur. The finding of two further cases within a few months of the initial observation suggests that with increasing awareness the murmur will be heard more often.

Once recognized, it is difficult to make an acceptable alternative diagnosis of the murmur. Its location and high frequency make it readily distinguish- 
able from the apical rumble of mitral stenosis, though this has been suggested as the main differential (Cheng, 1970b; Martin, Shaver, and Leonard, 1972). The sharp localization of the murmur and its crescendo-decrescendo quality make the diagnosis of aortic regurgitation unlikely.

The murmur is probably caused by turbulence of blood flow immediately distal to the stenosed segment. Turbulence is directly related to blood flow and vessel diameter, and is therefore unlikely to occur unless there is good flow. In neither of our patients in whom a stenosis was demonstrated angiographically was it considered to be greater than 50 per cent of the vessel lumen, suggesting that flow was not greatly reduced. Phasic diastolic coronary flow is reduced by experimental partial occlusion (Khouri, Gregg, and Lowensohn, 1967), so for a diastolic murmur to be heard the occlusion can only be slight. Further, a widely patent graft in the first patient gave no relief of symptoms, indicating that blood flow across the stenosed segment still had been adequate to supply that vessel's territory. No increase in overall coronary perfusion is obtained by grafting across a mild coronary obstruction in which the pre-existing distal pressures and flow are high (Smith et al., 1972).

Thus the diastolic murmur produced by stenosis of the left anterior descending coronary artery is a specific, easily recognizable murmur, and indicates only mild narrowing of that vessel. Because of the specific circumstances necessary for its production, and its physical characteristics, it is uncommon and easily missed, but when present it is a helpful aid in the diagnosis of coronary artery disease.

The authors wish to thank Mr. W. P. Cleland and his staff who performed the operations and Dr. Hugh
Baron, Dr. Sally Channon, and Dr. Wilfred Stokes who had kindly referred the patients.

\section{References}

Cheng, T. O. (I970a). Physical diagnosis of coronary artery disease. American Heart fournal, 80, 716.

Cheng, T. O. (1970b) Diastolic murmur caused by coronary artery stenosis. Annals of Internal Medicine, 72, 543.

Cohn, P. F., Vokonas, P. S., Williams, R. A., Herman, M. V., and Gorlin, R. (197I). Diastolic heart sounds and filling waves in coronary artery disease. Circulation, 44, 196.

Dock, W., and Zoneraich, S. (1967). A diastolic murmur arising in a stenosed coronary artery. American fournal of Medicine, 42, 617 .

Fearon, R. E., Cohen, L. S., O'Hara, J. M., and Goodyer, A. V. N. (I968). Diastolic murmurs due to two sequelae of atherosclerotic coronary artery disease: ventricular aneurysm and coronary artery stenosis. American Heart fournal, 76, 252.

Gregg, D. E. (1950). Coronary Circulation in Health and Disease. Henry Kimpton, London.

Gregg, D. E. (1967). The coronary circulation in the unanaesthetized dog. In Coronary Circulation and Erergetics of the Myocardium. Ed. by G. Marchetti and B. Taccardi. S. Karger, Basel.

Khouri, E. M., Gregg, D. E., and Lowensohn, H. S. (1967). Experimental coronary insufficiency. In Coronary Circulation and Energetics of the Myocardium. Ed. by G. Marchetti and B. Taccardi. S. Karger, Basel.

Lund-Larsen, P. B. (1967). Coronary artery stenosis murmur. Acta Medica Scandinavica, 182, 433.

Martin, C. E., Shaver, J. A., and Leonard, J. J. (1972). Physical signs, apexcardiography, phonocardiography, and systolic time intervals in angina pectoris. Circulation, 46, I098.

Sabiston, D. C., and Gregg, D. E. (1957). Effect of cardiac contraction on coronary blood flow. Circulation, 15, 14

Smith, S. C., Gorlin, R., Herman, M. V., Taylor, W. J., and Collins, J. J. (1972). Myocardial blood flow in man: effects of coronary collateral circulation and coronary artery bypass surgery. Fournal of Clinical Investigation, 51, 2556.

Requests for reprints to Dr. C. M. Oakley, Department of Medicine (Clinical Cardiology), Royal Postgraduate Medical School, Hammersmith Hospital, Du Cane Road, London W.I2. 